\title{
Modeling of Electricity Prices
}

\author{
Yunhe Hou, Member, IEEE, Yang He, Student Member, IEEE
}

\begin{abstract}
This is a summary of the presentation in the special session: "Digital Signal Processing for Green Power Systems and Delivery". The power sectors in various countries have established competitive markets for an industry that, for decades, consists of utility companies that own and operate generation, transmission and distribution systems. The objective of deregulation in power industry is to enhance the efficiency of power energy. With the liberalization of electric power industry, electricity price is widely recognized as a signal of electricity supply, consumption, and investment. Although the mechanisms that determine electricity prices are known, random events such as contingencies and congestive conditions of transmission lines can cause uncertainties. Therefore, it is difficult to develop analytical models for electricity prices that can be used by market or system planners and investors in their decision making process. The purpose of this paper is to introduce models of electricity prices that can be used by different market participants.
\end{abstract}

Index Terms - Electricity Prices, Stochastic Process, Time series

\section{INTRODUCTION}

E lectricity price, as a stochastic process, is widely recognized as a essential signal for power market operation and, ultimately, an efficient energy consumption [1]. The worldwide trend in liberalization of the electric power industry has introduced a competitive regime into power systems since the middle of 1990's. The aim of deregulation in power industry is to enhance the efficiency of electric energy production and distribution, increase quality and security of power systems. Unlike the situation in the regulated monopoly environment where there is a guaranteed rate of return, generation companies in the deregulated environment must take into account the market risks, especially the uncertainties caused by the fluctuation of electricity price in the market. As a result, accurate models of electricity prices are critical, both for suppliers and consumers [2].

The most significant distinction between power market and the regulated one is the mechanisms that determine electricity prices [3]. Electricity prices under the traditional structure are determined primarily by a cost recovery mechanism. Generally, electricity prices under the regulated regime are a function of load level, efficiency and availability of generating units, prices of fuel, and various operating constraints of generating units

The work is supported by Research Grant Council, Hong Kong SAR, under grants $717907 \mathrm{E}$ and $718608 \mathrm{E}$.

Yunhe Hou and Yang He are with the Department of Electrical and Electronic Engineering, The University of Hong Kong, Hong Kong, China (e-mail: ffwu@eee.hku.hk; yanghe@eee.hku.hk). and power grids, etc. By calculating all of factors, prices are established by regulatory agencies. The new competitive environment of the power industry led to a radical change on how prices of electricity are established. From economic point of view, the price of electricity in spot market is determined by the demand and supply for electricity. The electricity price will go up with increasing demand or decreasing supply. In power market operation, the cumulative supply curve is the results of bidding progress among different generation companies. Since the bidding strategy of each generation company is not transparent in the market, and the demand response changes the load level frequently, the market clear price will show a random characteristic.

Although the mechanisms that determine electricity prices are known, random events such as contingencies and congestive conditions of transmission lines, bidding strategies, and demand responses, can cause uncertainties. The real data in different power markets show that electricity prices vary with time and, furthermore, a traded commodity, its price has been displaying the highest level of volatility and the most complex features among all commodity prices. Stochastic processes are functions of time whose values at different times are random variables. Associated with the physical background of power market, different stochastic processes have been used to model electricity prices for various purposes.

\section{Characteristics OF EleCtricITY PRICES}

The essential issue for electricity price modeling is to understand its characteristics. This section addresses the challenge of capturing the general behavior of electricity prices, which are building blocks of electricity price models, no matter what kinds of mathematical tools are selected. Although, various police and rules are implemented in different markets, electricity prices share some general characteristics, especially, from an empirical point of view. More sophisticated analysis reveals that all of the general characteristics can be derived from the economic and physical fundamentals of electricity markets. In other word, a successful model should be built on the understanding of physical background, which might be illustrated from empirical experience.

The data used in this study consist of hourly electricity prices. Several markets around the world are examined. Fig. 1, Fig. 2 and Fig. 3 show, respectively, hourly prices of (1) PJM day-head electricity spot prices from 2002 to 2009, (2) New-England day-ahead electricity spot prices from Feb. 2003 to Feb. 2009, (3) . Nordic Pool system prices from 1996 to 2009. 


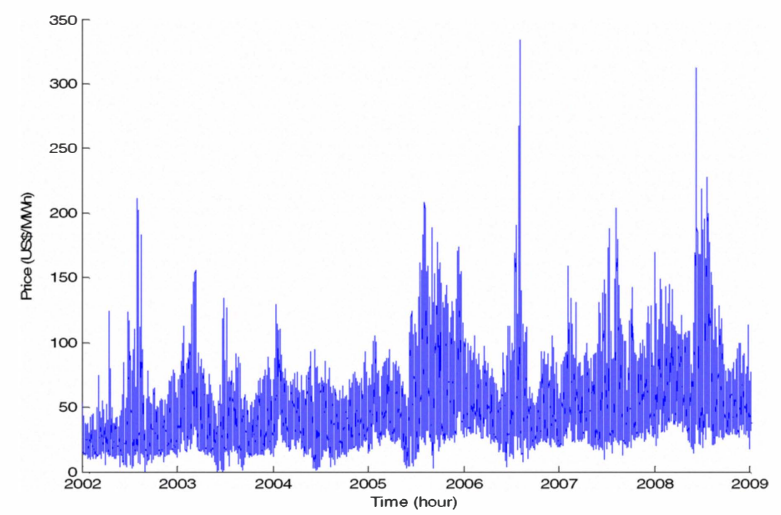

Fig. 1. PJM day-head electricity spot prices from 2002 to 2009

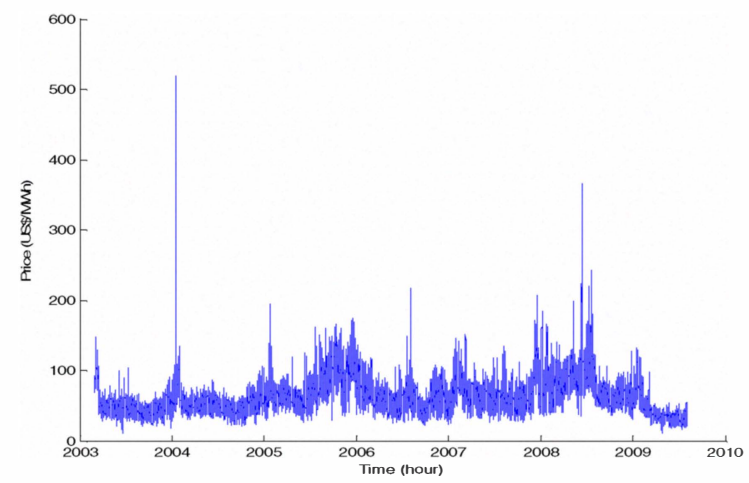

Fig. 2 New-England day-ahead electricity spot prices from Feb. 2003 to Feb. 2009

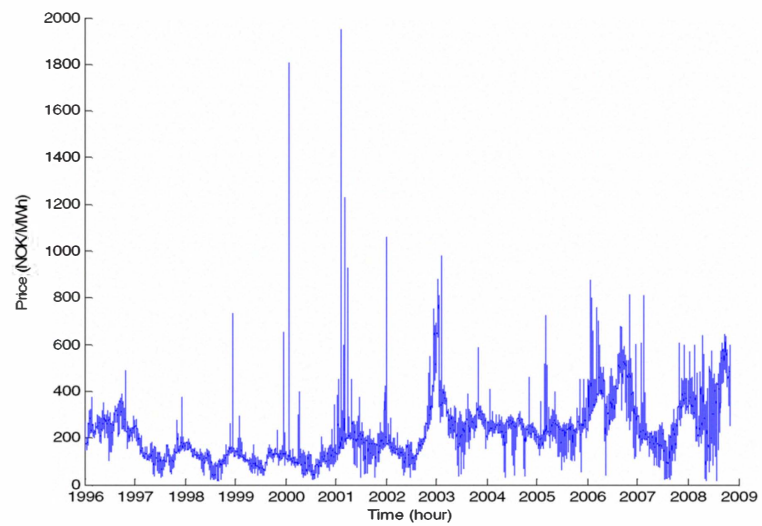

Fig. 3. Nordic Pool system prices from 1996 to 2009

The figures illustrate the cyclical and volatile nature of electricity prices in each market. Some properties of electricity are shown. First, electricity spot prices show a strong trend of mean reversion. This is a common feature in prices of other traded commodities [4]. The reason is that when the price of electricity is high, its supply tends to increase, putting a downward pressure on the price. Similarly, when the price is low, the supply of electricity tends to decrease, providing an upward lift to the price. Fig. 4 illustrates a sample of hourly price and quantity data for the time period from Aug 1, 1998 to Aug 22, 1998 in California. It is clear the trends of mean reversion for loads and electricity price. Furthermore, as a direct consequence of the corresponding seasonal fluctuations in demand, which arises from the changing conditions, i.e., temperature, generation outages and daylight, electricity price displays significantly seasonal property, as shown in Fig. 4.

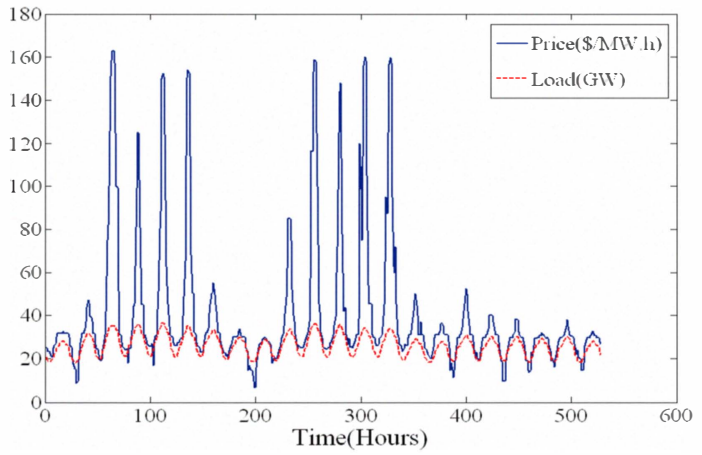

Fig. 4. Sample hourly electricity prices and load from Aug 1 to Aug 22, 1998, in California

The seasonal property of electricity spot prices is the next salient feature. It is known that electricity price is significantly affected by load level. As a direct consequence of the corresponding seasonal fluctuations in demand, which arises from the changing conditions, i.e., temperature, generation outages and daylight, electricity price displays significantly seasonal property, as shown in Fig. 4.

Empirically, strong stochastic volatilities or spikes are demonstrated by electricity prices in different power markets. Fig. 5 presents the histogram for the two months of PJM spot prices in summer 2000. Existence of the fat tails suggests that the probability of rare events is much higher than what is predicted by a Gaussian distribution. The implication is that the spikes in electricity data cannot be captured by simple Gaussian shocks. Future evidence can be found in [5]. From a point view of power market, the jump behavior of electricity spot prices is primarily due to the fact that a typical regional aggregate supply cost curve for electricity almost always has a kink at a certain capacity level and a steep upward slope beyond that capacity level. A forced outage of a major power plant or a sudden surge in demand will either shift the supply curve left or lift the demand curve up so that the regional electricity demand curve crosses the regional supply curve at its steep-rise portion, causing a jump in the price. When the contingency disappears in the short term, the high price will drop to its normal range, forming a spike.

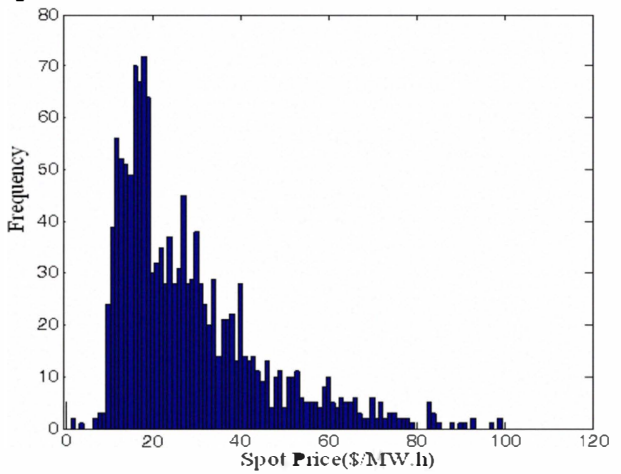

Fig. 5 Histogram for two months of PJM spot prices in summer 2000

It is known that electricity price is significantly depends on 
its cost and consumption, i.e., fuel cost and load level. The relationship between day-ahead hourly spot prices and real-time hourly electricity load in Pennsylvania-New Jersey-Maryland (PJM) market in 2008 is illustrated in Fig. 6. As illustrated in Fig. 6, generally, high load level results in expensive electricity price. The reason lies in the cost of a generating unit for peak load is higher than the one for base load. As a result, the fuel price also affects the electricity price. In the long-term, or low frequency, roughly, relationship between electricity price and fuel price is linear. The low frequency electricity price vs. fuel price is illustrated in Fig. 7.

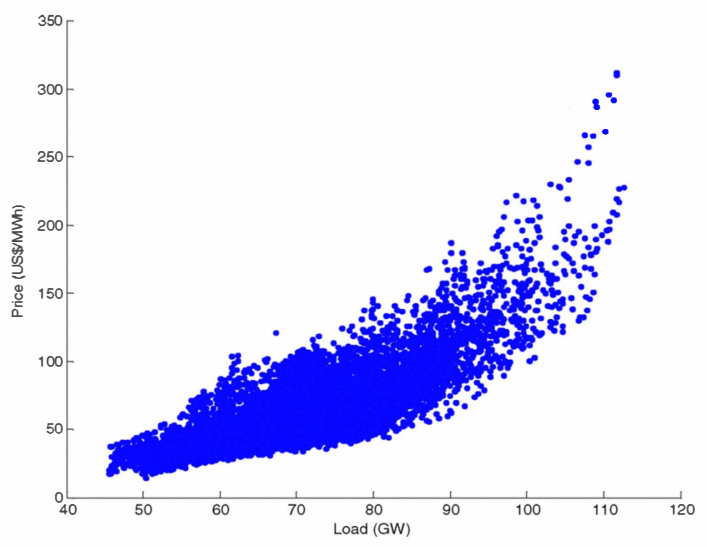

Fig. 6 Day-ahead hourly spot prices vs. real-time hourly electricity load

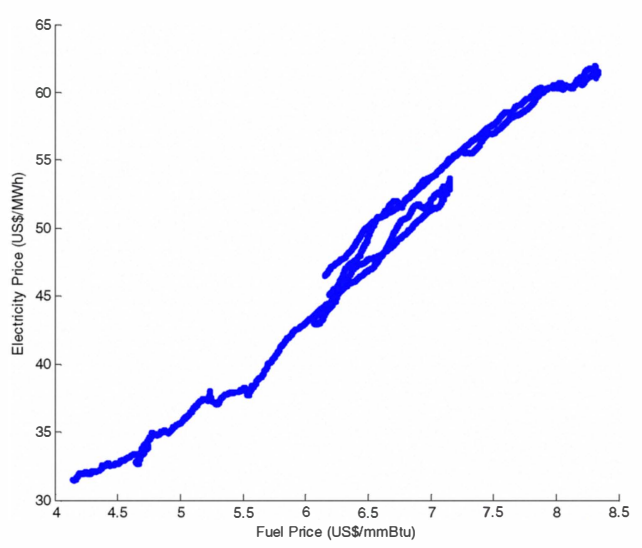

Fig. 7. Low-frequency electricity price vs. low frequency fuel price

To summarize, in order to construct a model for simulation of the electricity price process in the spot market, the following characteristics need to be considered:

- Strongly seasonal nature of prices;

- Mean reversion nature;

- Price dependent volatilities;

- Correlation between electricity price and other factors.

\section{Methodology of Electricity Price Modeling}

As a most essential issue of power market, large numbers of electricity price models have being established to meet the different requirements. Generally, these works can be roughly classified in three groups [6]: production costs models, continuous time stochastic models, and time series models.

Fundamental models focus on the mechanisms of electricity price established, not only production costing but also the impact of agents' strategic behavior on the market price. The concept entitled nodal price proposed by Schweppe is the pioneer of this group [3]. By this method, system conditions, biding of generation companies and consumers are fully considered. Recent work of this group involved more sophisticated analytical tools for realistic factors. The relationship between supply and demand is the basic consideration of this model. In supply-demand framework proposed by [7], the hourly day-ahead price of electricity is modeled. The level and probability of a spike in the spot price can be forecasted. Since real-time price elasticity of electricity contains important information on the demand response to the volatility of peak prices, [8] provides a quantification of the real-time relationship between total peak demand and spot market prices. A low value for the real-time price elasticity is found. The relationship between electricity price, volatility and electricity market structure of Singapore's electricity market is studied in [9]. Considering availability of the units and demand, different oligopoly models can be employed to analyze the mean and variance of electricity prices [10]. The essential physical characteristics of generating units, such as force outage rate[11], can be involved. The behaviors of prices under different policies [12] or different market clearing time [13] can be modeled by the models of this category. Furthermore, The impact of temperature [14], water temperatures [15] and information [16], can be studied in this framework as well.

The success of Geometric Brownian Motion in modeling of stock prices [4] leads to widely utilization of continuous time stochastic models of commodity prices. As a direct utilization of financial model, Geometric Brownian Motion is employed in early studies. Recently, a number of researchers, such as, Deng et al. [5, 17], Lucia and Schwartz [18] considered the use of mean reversion price models. To involved the jumps or spikes of the price, more sophisticated models are proposed [19, 20]. The basic idea is that deviations of the price from its equilibrium level are corrected and subjected to random perturbations. By these models, strongly seasonal nature of prices can be described. To deal with the jumps or spikes of electricity prices, [20] describe the dynamics of electricity price as a sum of non-Gaussian Ornstein-Uhlenbeck processes with jump processes, the normal variations and spike behavior of the prices are involved as well. Considering the relationship between explanatory variables such as load, hydro, thermal and nuclear resources available, and the electricity spot price through a dynamic regression model, price models can be seen as a switching model in which the system evolves through different states, where a particular dynamic regression model is adjusted in each one. Based on this idea, some papers, e.g. [21, 22], are focused on the regime switching characteristics of electricity prices.

As a signal with stochastic components, from a statistical point of view, time series models are used to study electricity price. The well known generalized autoregressive conditional heteroscedastic (GARCH) model is representative in this group. GARCH is used to describe electricity price volatility and the marginal cost of congestion on the NYISO market [23]. To deal with the fat tail, an exponential GARCH with extreme 
values is adopted in [24]. VAR Vector autoregression (VAR) allows regularities in the data to be studied without imposing as many prior restrictions as structural models impose, is used to describe price dynamics among 11 U.S. electricity spot markets[25]. Considering the mean-reverting characteristics of price, [26] employ the combined seasonal auto regressive integrated moving average (SARIMA) and discrete Markov processes to model prices of a power market.

\section{STRUCTURAL MODEL BASED ON SUPPLY AND DEMAND}

To derive analytical solution for investment evaluation and risk assessment, a model is purposed here. As mentioned in section II, series of characteristics are shared by electricity prices in different markets. These characteristics will be involved in this model.

Considering a stochastic process, $\tilde{x}(t)$, described by (1),

$$
d \tilde{x}(t)=k\left[\sum_{i=1}^{n} \alpha_{i}(t)-\tilde{x}(t)\right] d t+\sigma d \tilde{z}
$$

where $k$ and $\sigma$ are constants, $\alpha_{i}(t)$ is a deterministic function, $\tilde{z}$ is a white noise.

Denote

$$
E[\tilde{x}(t)]=\pi(t)
$$

Obviously, $E[\tilde{x}(t)]$ is the solution of following ordinary differential equation

$$
\frac{d \pi(t)}{d t}=k\left[\sum_{i=1}^{n} \alpha_{i}(t)-\pi(t)\right]
$$

Furthermore, $\pi(t)$ can be expressed as

$$
\pi(t)=\sum_{i=1}^{n} \pi_{i}(t)
$$

where $\pi_{i}(t)$ satisfies,

$$
\frac{d \pi_{i}(t)}{d t}=k\left[\alpha_{i}(t)-\pi_{i}(t)\right]
$$

Note that if $\alpha_{i}(t)$ is periodic, then $\pi_{i}(t)$ converges to a unique periodic oscillation.

First, if $k>0$, the solution of system (5) is unique.

Then, the solution of system (5) converges to a periodic oscillation. Let $\eta(\mathrm{t})$ be a solution of system (5). That is

$$
\frac{d \eta(t)}{d t}=k\left[\alpha_{i}(t)-\eta(t)\right]
$$

For time $t+T$, it is seen that

$$
\eta^{\prime}(t+T)=k\left[\alpha_{i}(t+T)-\eta(t+T)\right]=k\left[\alpha_{i}(t)-\eta(t+T)\right]
$$

where $T$ is the period of $\alpha_{i}(t)$.

Then $\eta(t+T)$ is a solution of system (5). Since the solution of system (5) is unique, it follows that : $\forall \varepsilon>0, \exists t_{1}>0$, when $t>t_{1}$

$$
|\eta(t+\omega)-\eta(t)|<\varepsilon
$$

This means that the solution of system (5) converges to a unique periodic oscillation. As a result, the multiple seasonality characteristics may be described by (1).

Another essential characteristic of electricity prices is jump or spike. Incorporating with the regime-switching method, model described by (1) can be used to describe the spikes. In this method, regime switches between a non-spike regime (where prices are unlikely to spike) and a spike regime. Most regime-switching models assume constant transition probabilities from one regime to another, e.g. from a non-spike to a spike regime. This simplification allows models to incorporate the magnitude and frequency of price spikes. For example, Fig. 8 shows scatter plots between demand (i.e., supply) levels and prices in the PJM electricity market from January 1, 1999 to Mar 31, 2002. Clearly, price spikes occur only when demand is high. In a competitive electricity market, the supply curve has an upward slope, reflecting the increasing marginal cost of power generation. When demand is low, the corresponding supply level is low so that the marginal cost of generation, or the price, is low. As demand increases, the marginal cost of supply increases, and as it approaches the limit of supply capacity, the marginal cost of generation rapidly increases, causing sudden and large price increases.

In this case, prices are unlikely to spike when demand is low compared to supply, but as the demand increases and approaches the supply capacity, prices are more likely to spike. Then, the transition probability from a non-spike to a spike regime cannot be constant. It should depend on the underlying supply and demand relationship.

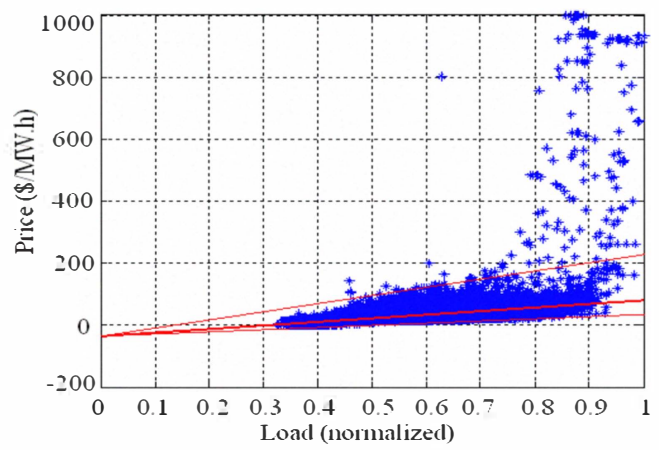

Fig. 8. PJM electricity market from January 1, 1999 to Mar 31, 2002

As an approximation, a hockey-stick shaped curve is shown in Fig. 9. The supply curve has this shape because of the increasing marginal cost, the limited capacity of electricity supply and the forced outage rate. In the short run, available power-generating facilities in the whole market are almost fixed. As demand increases, power companies increase their supply with facilities at higher marginal costs, but they cannot produce more electricity than the available capacities. As a result, near the limit of supply capacity, prices suddenly increase so that the slope of supply curve suddenly becomes steeper. Furthermore, the forced outage of generator increases this tendency. 


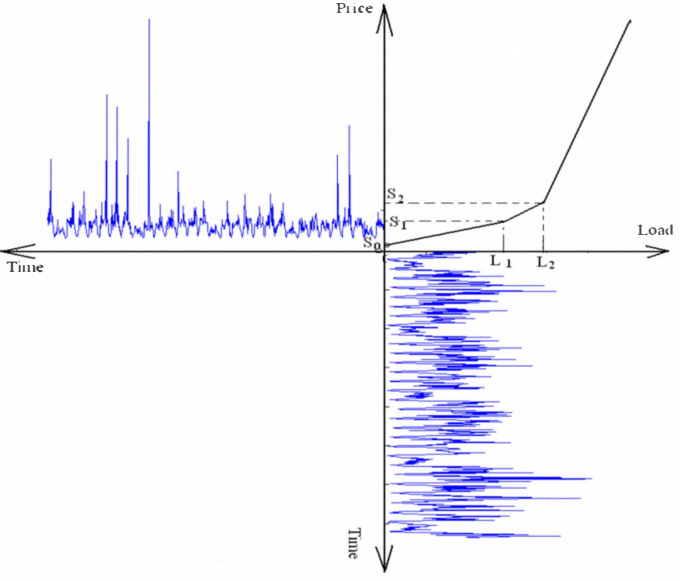

Fig. 9. A structural model based on supply and demand

The supply curve is modeled by two segments, i.e., base segment and spiking segment, as shown in Fig. 9. To simplify the problem, slopes of the segments are deterministic. Load is a stochastic process, i.e., a mean reversion process. The intersections of segments and the random demand settle the spot prices. Mathematically, this model can be written as

$$
\begin{array}{cc}
d \tilde{L}(t)=\eta[f(t)-\tilde{L}(t)] d t+v d \tilde{z} \\
\tilde{S}(t)=\alpha_{1} \tilde{L}(t)+b_{1} \quad 0<L \leq L_{1} \\
\tilde{S}(t)=\alpha_{2} \tilde{L}(t)+b_{2} & L_{1}<L
\end{array}
$$

where $a_{1}, a_{2}, b_{1}$ and $b_{2}$ are constants, $\eta$ is a parameter, $f(t)$ is the deterministic component of the load and $v$ is its volatility.

\section{CONCLUSION}

High efficiency energy consumption is not only depends on the development of technology, but also management. The transition of the power industry from a traditional utility company to a competitive electricity market has being implemented in power systems all over the world for about 20 years. In this new regime, the electricity price is the primary signal for all of participants. Strong volatility associated with uncertainty causes anxiety about price models. Large numbers of models for electricity price have been established to meet the requirements of different purposes. Models based on different stochastic processes are widely used in this area. However, most of these models focus on short-term price models, and only a few models can be employed to describe the behaviors of prices in middle-term. To implement green energy consumption, investments on energy resources are critical. Generation investment planning concerns future generator profit up to many years, an electricity price model for generation investment and scheduling, therefore, ought to capture the dynamics of electricity spot prices in a future time horizon of many years. Unfortunately, few available price models address this issue. The bottlenecks for the establishment of the long-term price model are: too many uncertainties involved in future prices, and lack of sufficient historical and forwards data.

\section{REFERENCES}

[1] D. Pilipovic, Energy risk: valuing and managing energy derivatives, 2ed ed. New York: The McGraw-Hill Companies 2007.

[2] R. Weron, Modeling and forecasting electricity loads and prices: a statistical approach. West Sussex, England: John Wiley\&Sons, Ltd, 2006.

[3] F. C. Schweppe, M. C. Caramanis, R. D. Tabors, and R. E. Bohn, Spot pricing of electricity: Boston: Kluwer Academic Publishers, 1988.

[4] J. Hull, Options, Futures, and Other Derivatives 7th Edition ed: Upper Saddle River, NJ, USA: Pearson Edition, Inc., 2008.

[5] S.-J. Deng and W. Jiang, "Levy process-driven mean-reverting electricity price model: the marginal distribution analysis," Decis. Support. Syst., vol. 40, no. 3-4, pp. 483-494, Oct. 2005.

[6] Z. Xia, "Pricing and risk management in competitive electricity markets," in School of Industrial and System Engineering, vol. Ph.D: Georgia Institute of Technology, 2005.

[7] A. Boogert and D. Dupont, "When supply meets demand: the case of hourly spot electricity prices," IEEE Trans. Power Syst., vol. 23, no. 2, pp. 389-398, May. 2008.

[8] M. G. Lijesen, "The real-time price elasticity of electricity," Energy Economics, vol. 29, no. 2, pp. 249-258, Sep. 2007.

[9] Y. Chang and C. Park, "Electricity market structure, electricity price, and its volatility," Economics Letters, vol. 95, no. 2, pp. 192-197, Mar. 2007.

[10] C. M. Ruibal and M. Mazumdar, "Forecasting the mean and the variance of electricity prices in deregulated markets," IEEE Trans. Power Syst., vol. 23, no. 1, pp. 25-32, Feb. 2008.

[11] C. L. Anderson and M. Davison, "A Hybrid System-Econometric Model for Electricity Spot Prices: Considering Spike Sensitivity to Forced Outage Distributions," IEEE Trans. Power Syst., vol. 23, no. 3, pp. 927-937. 2008.

[12] T. S. Genc and S. Sen, "An analysis of capacity and price trajectories for the Ontario electricity market using dynamic Nash equilibrium under uncertainty," Energy Economics, vol. 30, no. 1, pp. 173-191, Jan. 2008.

[13] J. Nutaro and V. Protopopescu, "The Impact of Market Clearing Time and Price Signal Delay on the Stability of Electric Power Markets," IEEE Trans. Power Syst., vol. 24, no. 3, pp. 1337-1345. 2009.

[14] P. M. Schwarz, T. N. Taylor, M. Birmingham, and S. L. Dardan, "Industrial response to electricity real-time prices: short run and long run," Economic Inquiry, vol. 40, no. 4, pp. 597-610, Oct. 2002.

[15] A. Boogert and D. Dupont, "The nature of supply side effects on electricity prices: The impact of water temperature," Economics Letters, vol. 88, no. 1, pp. 121-125, Jul. 2005.

[16] T. Ishikida and P. P. Varaiya, "Pricing of electric power under uncertainty: information and efficiency," IEEE Trans. Power Syst., vol. 10, no. 2, pp. 884-890, May. 1995.

[17] S. J. Deng, B. Johnson, and A. Sogomonian, "Exotic electricity options and the valuation of electricity generation and transmission assets," Decis. Support. Syst., vol. 30, no. 3, pp. 383-392. 2001.

[18] J. J. Lucia and E. Schwartz, "Electricity prices and power derivatives: Evidence from the Nordic power exchange," Review of Derivatives Research, vol. 5, no. 1, pp. 5-50. 2002.

[19] N. K. Nomikos and O. Soldatos, "Using affine jump diffusion models for modelling and pricing electricity derivatives," Applied Mathematical Finance, vol. 15, no. 1, pp. 41-71, Mar. 2008.

[20] F. E. Benth, J. Kallsen, and T. Meyer-Brandis, "A non-Gaussian Ornstein-Uhlenbeck process for electricity spot price modeling and derivatives pricing," Applied Mathematical Finance, vol. 14, no. 2, pp. 153-169, May. 2007.

[21] P. Kosater and K. Mosler, "Can Markov regime-switching models improve power-price forecasts? Evidence from German daily power prices," Appl. Energ., vol. 83, no. 9, pp. 943-958. 2006.

[22] N. Haldrup and M. O. Nielsen, "A regime switching long memory model for electricity prices," J. Econometrics., vol. 135, no. 1-2, pp. 349-376, Aug. 2006.

[23] L. Hadsell and H. A. Shawky, "Electricity price volatility and the marginal cost of congestion: An empirical study of peak hours on the NYISO market, 2001-2004," Energy J., vol. 27, no. 2, pp. 157-179. 2006.

[24] F. K. Chan and P. Gray, "Using extreme value theory to measure value-at-risk for daily electricity spot prices," International Journal of Forecasting, vol. 22, no. 2, pp. 283-300, Mar. 2006.

[25] H. Park, J. W. Mjelde, and D. A. Bessler, "Price dynamics among U.S. electricity spot markets," Energy Economics, vol. 28, no. 1, pp. 81-101, Nov. 2006. 
[26] M. Olsson and L. Soder, "Modeling real-time balancing power market prices using combined SARIMA and Markov processes," IEEE Trans. Power Syst., vol. 23, no. 2, pp. 443-450, May. 2008.

Yunhe Hou (M' 06) received the B.E (1999), M.E(2002) and Ph.D(2005) degrees from the Huazhong University of Science and Technology, China. He worked as a postdoctoral research fellow at Tsinghua University from 2005 to 2007. He was a visiting scholar at Iowa State University, Ames, and a researcher of University College Dublin, Ireland from 2008 to 2009. He is currently with the University of Hong Kong, Hong Kong, as a research assistant professor.

Yang He (S'09) received his B.E. (2005) from Zhejiang University, Hangzhou, China. Since September 2005, he has been with the University of Hong Kong, pursuing a $\mathrm{Ph} . \mathrm{D}$. degree. His interest is in electricity price modeling and generation investment planning. 